\title{
Clinical characteristics of struma ovarii
}

\author{
Seung-Chul Yoo, Ki-Hong Chang, Mi-Ok Lyu, Suk-Joon Chang, Hee-Sug Ryu, Haeng-Soo Kim \\ Department of Obstetrics and Gynecology, Ajou University School of Medicine, Suwon, Korea
}

\begin{abstract}
Objective: To evaluate the clinical characteristics of struma ovarii.
Methods: Twenty-five cases of struma ovarii were reviewed retrospectively from June 1994 to April 2007. The presenting clinical, radiologic, and pathologic features of the patients were reviewed.

Results: The mean age of the patients in this study was 45.3 years. The majority was of premenopausal status. Sixteen patients had clinical symptoms such as low abdominal pain, palpable abdominal mass and vaginal bleeding. Although one patient had an abnormal thyroid function test, the laboratory findings normalized after operative treatment. CA-125 levels were elevated in 6 cases. Diagnosis by preoperative imaging studies were 8 dermoid cysts, while only 3 cases were diagnosed as struma ovarii. There were 4 cases of malignant struma ovarii, and no patients with recurrent disease.

Conclusion: Struma ovarii is a rare tumor. The presented clinical, laboratory and radiological findings of patients are very diverse. The diagnosis was confirmed by pathologic findings. The treatment of benign struma ovarii is surgical resection only. The cases of malignant struma ovarii may need adjuvant treatment, but recurrence is uncommon.
\end{abstract}

Key Words: Struma ovarii, Dermoid tumor, Malignancy

\section{INTRODUCTION}

Struma ovarii is a variant of dermoid tumors of the ovary in which thyroid tissue components is the major constituent. ${ }^{1}$ Thyroid tissue is observed not uncommonly in $5-15 \%$ of dermoid tumors, but to qualify as a struma ovarii tumor the thyroid proportion must comprise more than $50 \%$ of the overall tissue. $^{2}$

Struma ovarii of the ovary is a relatively rare tumor which comprises $1 \%$ of all ovarian tumors and $2.7 \%$ of all dermoid tumors. ${ }^{3}$ This tumor was first described in 1889 by Boettlin, who observed the presence of thyroid follicular tissue in ovaries, and further reports thereafter were published by Gottschalk. However, due to rarity of this type of tumor there has been a paucity of data in the past literature pertaining to diagnosis and treatment of this tumor. ${ }^{4,5}$

The authors of this study report our experience of a total of 25 patients with struma ovarii who received surgical treatment at our institution, and attempt to delineate the clinical features and characteristics of this tumor with respect

Received February 25, 2008, Revised March 21, 2008,

Accepted June 9, 2008

\section{Address reprint requests to Ki-Hong Chang}

Department of Obstetrics and Gynecology, Ajou University School of Medicine, San-5, Woncheon-dong, Yeongtong-gu, Suwon 443721, Korea

Tel: 82-31-219-5247, Fax: 82-31-219-5245

E-mail: cpobgy@hanmail.net to ultrasonographic findings, histological characteristics, and prognosis after treatment.

\section{MATERIALS AND METHODS}

This study was conducted from June 1st 1994 to April 30th 2007, and included 25 patients with histologically confirmed (benign and malignant) struma ovarii whose medical records were retrospectively reviewed and analysed.

The medical records of the above patients were reviewed with respect to age, obstetrical history, and clinical symptoms such as abnormal vaginal bleeding or lower abdominal pain, symptoms related to increased thyroid function, pre-operative imaging studies, serum CA-125 levels, post-operative histological diagnosis, and prognosis.

\section{RESULTS}

The clinical characteristics of the 34 patients are shown in Table 1 . The most common presenting symptoms at the time of diagnosis were lower abdominal pain $(n=7,20.6 \%)$, followed by palpable lower abdominal mass $(n=8,23.5 \%)$. The next common symptom was abnormal vaginal bleeding $(n=3,8.8 \%)$. However, no definite presenting symptoms were declared in the remaining 14 patients $(41.2 \%)$, and the presence of an ovarian tumor was an incidental finding by ultrasonography (Table 1).

Pre-operative radiologic imaging studies such as ultra- 
Table 1. Patient characteristics $(n=34)$

\begin{tabular}{lcr}
\hline \multicolumn{1}{c}{ Characteristic } & No. of patients & $\%$ \\
\hline Age distribution & & \\
$21-30$ & 6 & 17.6 \\
$31-40$ & 8 & 23.5 \\
$41-50$ & 9 & 26.5 \\
$51-60$ & 4 & 11.8 \\
$61-70$ & 4 & 11.8 \\
$71-80$ & 3 & 8.8 \\
Initial symptoms & & \\
Abdominal pain & 7 & 20.6 \\
Palpable mass/abdominal distension & 8 & 23.5 \\
Vaginal bleeding & 3 & 8.8 \\
No specific symptom & 14 & 41.2 \\
Others & 2 & 5.9 \\
\hline
\end{tabular}

sonography was able to diagnose struma ovarii in only $4 / 34$ $(11.8 \%)$ patients. Other pre-operative diagnosis which was later confirmed to be struma ovarii were dermoid tumors in $7 / 34(20.6 \%)$ patients, benign cysts in $3 / 34(8.8 \%)$, and endometriomas in $4 / 34$ (11.8\%). Struma ovarii tumors that appeared as malignant tumors were observed in 10/34 (29.4\%) patients (Table 2 ).

Surgical therapy was conducted in all 34 patients. Surgical modalities consisted of total hysterectomy with unilateral or bilateral salpingo-oophorectomy in 19/34 (55.9\%) patients, unilateral salpingo-oophorectomy only in $8 / 34$ (23.5\%) patients, and extirpation of struma ovarii in 7/34 (20.6\%) patients. All surgeries were by exploratory laparotomy or laparoscopic methods, and among the 2 patients confirmed with malignant struma ovarii, infra-colic omentectomy was also performed.

Malignant struma ovarii was present in 5 of the 34 (14.7\%) patients. Four patients were stage Ia disease and therefore did not receive any adjuvant therapy, while the remaining one patient was stage IIIc disease and subsequently underwent 6 cycles of chemotherapy. This patient has been followed up for 4 years and is as yet free of disease.

There were 3 patients with tachycardia but no other manifestations of hyperthyroid function such as unexplained weight loss that suggested true thyroid hyperfunction. Serum CA-125 levels were examined in 13 patients ( 3 malignant and 10 benign), and were observed to be elevated in 4 of these patients, which all normalized after surgical therapy.

In this present study there were 4 patients with ascites.

Only those patients with malignant struma ovarii were followed up, for a median of 65 months (52-81 months).

\section{DISCUSSION}

Struma ovarii comprises $2.7 \%$ of all dermoid tumors of the ovary, and very rarely presents in a malignant form, occurring in $0.3 \%$ to $5 \%$ of all struma ovarii tumors. ${ }^{6,7}$
Table 2. Preoperative image study findings (transvaginal sonography, $\mathrm{n}=34$ )

\begin{tabular}{lcr}
\hline Preoperative diagnosis & No. of patients & $\%$ \\
\hline Struma ovarii & 4 & 11.8 \\
Dermoid cyst & 7 & 20.6 \\
Endometrioma & 4 & 11.8 \\
Other benign cyst & 3 & 8.8 \\
Malignancy & 10 & 29.4 \\
No diagnosis* & 6 & 17.6 \\
\hline
\end{tabular}

*ovarian mass was incidentally found in 6 patients during the surgery and the diagnosis was made after surgery

In our institution, the medical records showed that among the total of 10, 442 patients who visited our Department of Obstetrics and Gynecology for evaluation of ovarian tumors, 706 patients were diagnosed with dermoid tumors. Therefore, the 34 struma ovarii patients represented $4.8 \%$ of all dermoid patients, among whom $14.7 \%$ (5/34) were malignant struma ovarii. These figures are markedly different from previously reported data, particularly the high incidence of malignant struma ovarii, and which may be attributed to the rarity of struma ovarii and hence the very low number of previous study reports.

Clinical symptoms that may manifest due to the presence of a struma ovarii are lower abdominal pain, palpable lower abdominal mass, abnormal vaginal bleeding, ascites, hydrothorax, elevated thyroid function, and rarely thyroid tumors. ${ }^{8,9}$ Previous reports have shown that patients with struma ovarii are largely without symptoms, or are accompanied by non-specific symptoms that are similar to other ovarian neoplasms. ${ }^{10}$ The results of this study seem to be in agreement with the above observations as demonstrated by the fact that in 14 of the 34 patients, there were no presenting symptoms, and the tumors were discovered during routine check-up ultrasound studies. When symptoms were present, the majorities were non-specific, consisting of lower abdominal pain, palpable lower abdominal mass, and abnormal vaginal bleeding. It has been recommended in a previous report that thyroid function studies be conducted in the presence of symptoms and signs related to thyroid hyperfunction. ${ }^{11}$ In our 34 cases, there were no patients with overt hyperthyroid symptoms and therefore thyroid function profiles were not carried out. It cannot be fully explained why there were no overt cases with thyroid hyperfunction with our group of patients. The incidence of such thyroid hyperfunction has been reported to be $5-8 \%$ of patients with struma ovarii. $^{12,13}$

The occurrence of ascites in patients with struma ovarii has been observed to be vary, ranging $17-33.3 \%$ according to several authors. ${ }^{1,3,14}$ The precise mechanisms of the formation of ascites in struma ovarii patients are unclear to date, but it has been suggested that ascites, if present, usually regresses 
spontaneously after surgical removal of struma ovarii.

The widely accepted tumor marker of ovarian cancers, CA-125, is found to be increased in $80 \%$ of epithelial ovarian carcinomas. This marker is also elevated in other tumorous lesions of the endometrium, intestines, breasts, and lungs, as well as in non-malignant related gynecologic conditions, thus indicating that there is a limit to the clinical application of CA-125 as a tumor marker or malignant ovarian neoplasms. Moreover, it has been postulated that increased levels of CA-125 is not a direct consequence of the presence of a malignant tumor, but rather a secondary effect due to the presence of ascites. ${ }^{15}$ We observed in our study that CA-125 was increased in 4 among 13 patients ( 3 malignant and 10 benign) in whom CA-125 was examined, and only among 1 of the 3 patients with malignant struma ovarii. In the latter group, the degree of elevation was minimal, and as CA-125 was also found to be elevated in 3/10 patients with benign struma ovarii, it is proposed that serum CA-125 measurements are of little clinical value in struma ovarii patients.

The diagnostic criterion of malignant struma ovarii is based on histological features of the resected ovary. Although not yet uniformly standardized, these criteria include cellular atypia and increased hyperplasia, nuclear pleomorphism, mitotic activity, and vascular and/or capsular invasion. ${ }^{8,16}$ Distant metastasis has been reported to be a very uncommon feature of struma ovarii, and even if present, has a protracted course. An extreme case where distant metastasis was discovered 26 years after initial diagnosis has been reported. ${ }^{1}$ Sites of common metastases are the omentum, mesentery, and lymph nodes via the peritoneum, and the liver, brain, bone, lungs, and the contralateral ovary via the hematogenous route. $^{16,17}$

Therapy for benign struma ovarii is surgical resection. All patients in our study underwent either extirpation of the tumor or salpingo-oophorectomy with or without hysterectomy, and no patient showed recurrent disease. Due to the rarity of malignant struma ovarii, there is a paucity of data in the past literature regarding the optimal treatment modality for such patients. For women desiring further child-bearing, unilateral salpingo-oophorectomy may be a feasible option in the absence of capsular invasion or distant metastasis. ${ }^{6}$ Although infrequent, there have been reports of cases where women have had successful pregnancies after such conservative procedures. ${ }^{18}$ For advanced stage disease the surgical protocol is the same as for epithelial ovarian cancers. A recent proposal has been put forward in which surgical resection of tumor is accompanied by radioiodine therapy, but this has not been further substantiated. ${ }^{19}$ Among the 5 patients with malignant struma ovarii in this study, only 1 patient was with advanced disease stage IIIc. The other remaining 4 patients did not demonstrate any extension beyond the capsule, and were thus treated with simple hysterectomy with or without salpingo-oophorectomy, without adjuvant therapy. All 5 patients have been free of recurrent disease up to the last follow-up.

An adjuvant treatment modality that has been suggested for residual disease or metastatic/recurrent disease is radioiodine therapy, which has been reported to result in favorable outcomes. ${ }^{2}$ For patients with multiple metastatic lesion or those who do absorb radioiodine poorly, external beam radiation has been proposed. ${ }^{16}$ In addition, the role of iodine scans have been emphasized in the detection of recurrent disease after termination of therapy. ${ }^{20}$

In summary, the review of struma ovarii patients treated at our institution shows that this disease is difficult to diagnose on the basis of clinical manifestations or imaging studies as the presenting clinical features are widely diverse. Surgical treatment as for dermoid tumors is sufficient for benign lesions, and even if secondary conditions such as thyroid hyperfunction, ascites, or hydrothorax are present, these usually regress spontaneously upon surgical removal of the primary tumor. On the other hand, the optimal treatment modality for malignant struma ovarii is as yet unclear and remains to be established.

\section{REFERENCES}

1. Kempers RD, Dockerty MB, Hoffmann DL, Bartholomew LG. Struma ovarii: Ascitic, hyperthyroid and asymptomatic syndromes. Ann Intern Med 1970; 72: 883-93.

2. Willemse PH, Oosterhuis JW, Aalders JG, Piers DA, Sleijfer DT, Vermey A, et al. Malignant struma ovarii treated by ovariectomy, thyroidectomy, and 131 I administration. Cancer 1987; 60: 178-82.

3. Kim SJ, Pak K, Lim HJ, Yun KH, Seong SJ, Kim TJ, et al. Clinical diversity of struma ovarii. Korean J Obstet Gynecol 2002; 45: 748-52.

4. Boettlin R. Uber zahnentwickelung in dermoid cysten des ovariums. Virchows Arch Path Arat 1889; 115: 493-504.

5. Gottschalk S. Ein neuer typus einer kleincystischen bosartigen eierstockgeschwulst. Arch Gynak 1899; 59: 676-98.

6. Gould SF, Lopez RL, Speers WC. Malignant struma ovarii. A case report and literature review. J Reprod Med 1983; 28: 415-9.

7. Teilum G. Struma ovarii. In: Teilum G, editor. Special Tumors of Ovary and Testis. Philadelphia: J.B. Lippincott; 1971. p.166.

8. Bhansali A, Jain V, Rajwanshi A, Lodha S, Dash RJ. Follicular carcinoma in a functioning struma ovarii. Postgrad Med J 1999; 75: 617-8.

9. Zalel Y, Seidman DS, Oren M, Achiron R, Gotlieb W, Mashiach $\mathrm{S}$, et al. Sonographic and clinical characteristics of struma ovarii. Ultrasound Med 2000; 19: 857-61.

10. March DE, Desai AG, Park CH, Hendricks PJ, Davis PS. Struma ovarii hyperthyroidism in a postmenopausal women. J Nucl Med 1998; 29: 263-5.

11. Jang KH, Kim YT, Ryu HS, Kwon HC, Lee EJ, Lee HC, et al. Clinical diversity of struma ovarii. Korean J Obstet Gynecol 1997; 40: 1683-9.

12. Ayhan A, Yanik F, Tuncer R, Tuncer ZS, Ruacan S. Struma ovarii. Int J Gynaecol Obstet 1993; 42: 143-6.

13. Marcus CC, Marcus SL. Struma ovarii. A report of 7 cases and a review of the subject. Am J Obstet Gynecol 1961; 81: 752-62.

14. Smith FG. Pathology and physiology of struma ovarii. Arch Surg 1946; 53: 603-26. 
15. Leung YC, Hammond IG. Limitation of CA 125 in the preoperative evaluation of a pelvic mass: Struma ovarii and ascites. Aust NZ J Obstet Gynaecol 1993; 33: 216-7.

16. O'Connell ME, Fisher C, Harmer CL. Malignant struma ovarii: Presentation and management. Br J Radiol 1990; 63: 360-3.

17. Pardo-Mindan FJ, Vazquez JJ. Malignant struma ovarii: Light and electrion micrdscopic study. Cancer 1983; 51: 337-43.

18. Ihalagama IR, Hewavisenthi SJ, Wijesinghe PS. Pregnancy following treated malignant struma ovarii. Ceylon Med J 2004; 49:
90-1.

19. DeSimone CP, Lele SM, Modesitt SC. Malignant struma ovarii: A case report and analysis of cases reported in the literature with focus on survival and I131 therapy. Gynecol Oncol 2003; 89: 543-8.

20. Sun LQ, Zhou FN, Guo LH. Struma ovarii: Analysis of a series of 9 cases and review of the literature. Int J Gynecol Cancer 2006; 16: 698 . 